\title{
Real-time control for robotic hand application based on pressure sensor measurement
}

\begin{abstract}
This paper presents the use of data acquisition (DAQ) device and LabVIEW program to evaluate and measure the pressure distribution for real-Time robotic hand applications. An analog conditioning circuit has been implemented to calibrate and extract data from the utilized sensors to be processed by the DAQ and LabVIEW. Furthermore, an adequate signal processing analysis is performed on acquired data to investigate the desired measurements of pressure distribution for the robotic hand control purpose. This framework is considered as one of the techniques that can be used for robotic hand applications based on pressure sensor distribution and real-Time monitored through the LabVIEW.
\end{abstract}

Keyword: DAQ; Pressure distribution; Pressure sensor; Robotic hand; Signal processing 